\title{
Evaluating the impact of the genitourinary multidisciplinary tumour board: Should every cancer patient be discussed as standard of care?
}

Kyle Scarberry ${ }^{1,2}$; Lee Ponsky ${ }^{1,2}$; Edward Cherullo, ${ }^{1,2}$; William Larchian ${ }^{1,2}$; Donald Bodner ${ }^{1,2}$; Matthew Cooney ${ }^{2,3}$; Rodney Ellis ${ }^{2,4}$; Gregory MacLennan ${ }^{2,5}$; Ben Johnson ${ }^{1,2}$; William Tabayoyong ${ }^{1,2}$; Robert Abouassaly ${ }^{1,2}$

${ }^{1}$ Urology Institute, University Hospitals Cleveland Medical Center; ${ }^{2}$ Case Western Reserve University School of Medicine; ${ }^{3}$ Division of Hematology/Oncology, University Hospitals Cleveland Medical Center; ${ }^{4}$ Department of Radiation Oncology, University Hospitals Cleveland Medical Center; ${ }^{5}$ Department of Pathology, University Hospitals Cleveland Medical Center; Cleveland, OH, United States

Cite as: Can Urol Assoc J 2018 May 14; Epub ahead of print. http://dx.doi.org/10.5489/cuaj.5150

Published online May 14, 2018

$* * *$

\section{Abstract}

Introduction: We sought to prospectively evaluate the effectiveness of the multidisciplinary tumour board (MTB) on altering treatment plans for genitourinary (GU) cancer patients. Methods: All GU cancer patients seen at our tertiary care hospital are discussed at MTB. We prospectively collected data on adult patients discussed over a continuous, 20-month period. Physicians completed a survey prior to MTB to document their opinion on the likelihood of change in their patient's treatment plan. Logistic regression was used to asses for factors associated with a change by the MTB, including patient age or sex, malignancy type, the predicted treatment plan, and the provider's years of experience or fellowship training. Results: A total of 321 cancer patients were included. Patients were primarily male (84.4\%) with a median age of 67 (range 20-92) years old. Prostate (38.9\%), bladder (31.8\%), and kidney cancer (19.6\%) were the most common malignancies discussed. A change in management plan following MTB was observed in 57 (17.8\%) patients. The physician predicted a likely change in six $(10.5 \%)$ of these patients. Multivariate logistic regression did not determine physician prediction to be associated with treatment plan change, and the only significant variable identified was a plan to discuss multiple treatment options with a patient (odds ratio 2.46; 95\% confidence interval 1.09-9.54).

Conclusions: Routine discussion of all urologic oncology cases at MTB led to a change in treatment plan in $17.8 \%$ of patients. Physicians cannot reliably predict which patients have their 
treatment plan altered. Selectively choosing patients to be presented likely undervalues the impact of a multidisciplinary approach to care.

\section{Introduction}

Multidisciplinary coordination of care is a requirement for Commission on Cancer accreditation ${ }^{1}$ and National Comprehensive Cancer Network (NCCN) guidelines on prostate cancer recommend utilizing a multidisciplinary tumour board (MTB) to address challenging cases ${ }^{2}$. There are many proposed benefits to such MTB meetings, including evidence-based decision making, improved accuracy in diagnosis, increased access to clinical trials, improved communication between physicians of different specialties, cost-effective care, clinician education, and improved patient and clinician confidence and satisfaction ${ }^{3-7}$. Despite the wideadoption of MTBs in oncology, there is a lack of evidence regarding which cancer patients may benefit from discussion at MTBs.

Many specialties have established the usefulness of multidisciplinary tumour boards, with decisions on treatment recommendations being routinely altered by a MTB conference discussion in gynecologic cancer ${ }^{5}$, head and neck cancer ${ }^{6}$, breast cancer ${ }^{8}$, and upper gastrointestinal tract cancer ${ }^{9}$ patients. There is discrepancy in the literature about the effectiveness of MTBs in the field of urology. One study reports only $1.6 \%$ of genitourinary tract (GU) cancer patients experienced a change in their treatment decision following MTB discussion $^{10}$, while others report rates as high as $26.7-32.3 \%{ }^{3,11}$. As such, current NCCN guidelines limit recommendations to only select GU oncology patients ${ }^{2}$.

We hypothesized that GU MTB discussion results in significant treatment plan changes for GU oncology patients, and that physicians cannot predict which patients' management plans change from inclusion in MTB discussion.

\section{Methods}

The Genitourinary Tumour Board at University Hospitals Cleveland Medical Center is a 1-hour weekly meeting of physicians and nurses from urology, radiation oncology, medical oncology, radiology, and pathology to review and discuss every genitourinary cancer patient seen by physicians in the healthcare system. Each patient's history, including their pathologic and/or radiologic diagnoses, is reviewed by the group with all members of the different subspecialties present.

From September 2011 to April 2013, physicians were asked to complete a two-question survey for each patient they were to present at the following week's tumour board meeting. The questionnaire asked "What is your treatment plan for this patient?" and "How likely do you think this plan is to change following tumour board discussion?”, with options for the latter question as follows: (1) Very Unlikely; (2) Unlikely; (3) Likely; (4) Very Likely. Survey results, treatment decision determined by tumour board consensus, and patient demographic information were recorded by a research assistant at each meeting. 
Patients over 18 years of age with a primary diagnosis of a genitourinary malignancy were included in the study if the presenting physician responded to the survey and a consensus treatment decision was made by the tumour board. Cancer types were classified as prostate cancer, kidney cancer, bladder cancer, testicular cancer, upper tract urothelial cancer, or "other" (e.g. penile, adrenal) for analysis purposes. Patients were often presented to the MTB more than once, such as when new pathology was available after surgical treatment, with each instance recorded as a separate patient for data analysis purposes. Additional data collected included patient age and gender, as well as provider specialty, fellowship training in urologic oncology, and number of years in practice.

Treatment plans were categorized as "surgery", "radiation therapy”, "medical therapy", or "multimodal therapy" based on survey responses with an additional "discuss options" category included, in which physicians planned to offer more than one treatment option to a patient. A plan of "surveillance" was designated to patients offered active surveillance" such as in patients with low risk prostate cancer, or in patients who will continue surveillance of their disease after definitive treatment with laboratory testing, follow-up, and/or imaging. Patients who require further imaging or labs before treatment decisions could be made were classified as "further workup". Infrequent answer choices including "hospice care" and "clinical trial" were grouped together with "multimodal therapy" and "further workup" in the category of "other" for statistical analysis. Patients were classified as having a change in their management plan if a significant change in overall treatment modality (e.g., radiation therapy to surgery) or an addition of a treatment modality (e.g., surgery to chemotherapy and surgery) was observed.

Bivariate associations were tested with the Pearson chi-square and the two-sided t-test, as appropriate. Multiple logistic regression was performed to evaluate associations between multiple patient and physician characteristics, including the physician predicted likelihood of change in management plan, in order to determine factors associated with a change in treatment plan following MTB discussion. Stata SE, version 13.0 (StataCorp, College Station, TX) was used to perform all statistical analyses. A p-value of $<0.05$ was used to determine statistical significance.

\section{Results}

During the study period, 321 patients were discussed at the MTB with the physician's treatment plan and opinion on change likeliness documented prior to the meeting. The patients were primarily male (82.2\%) with a median patient age of 67 (range 20-92) years old. The most common diagnoses were prostate (38.9\%), bladder (31.8\%), kidney (19.6\%), upper tract urothelial carcinoma (3.7\%), and testicular (2.5\%) cancers. As shown in Table 1, 57 patients (17.8\%) experienced a change in treatment decision following MTB discussion. Among all patients, physicians predicted that treatment would be "very likely" to change in $0.0 \%$ of all cases, "likely" to change in $9.0 \%$, "unlikely" to change in $45.2 \%$, and "very unlikely" to change 
in $45.8 \%$. Of the 57 patients who experienced a change in treatment plan, 6 (10.5\%) were predicted by the physician to be "likely" to change. Of the 264 patients who did not have a change in plan, 23 (8.7\%) were determined to be "likely" to have a change.

Table 2 reveals that patient age, gender, cancer type, provider clinical experience or training, and provider prediction of the likelihood of change were not associated with a change in management following MTB discussion on univariate or multivariate logistic regression analysis. A pre-MTB physician treatment plan of “discuss options” (OR 2.46, 95\% CI 1.09-9.54) was associated with a change in management plan following MTB meeting on multivariate logistic regression.

The types of management changes are characterized in Table 3. Of the management changes observed, the majority were from a single modality treatment to a different treatment in 23 patients (40.4\%), with a change from "discuss options" or "surveillance" to "medical therapy" being the most commonly observed changes. For ten patients (17.5\%), presenting physicians felt their disease warranted treatment but MTB consensus favored surveillance. A more intensive management approach was favored by the MTB consensus in 17 patients, with physicians favoring surveillance in 15 (26.3\%) patients planned who would ultimately be recommended treatment and 2 (3.5\%) patients recommended multimodal therapy after being thought to warrant only single modality therapy prior to MTB discussion. Only two patients in the study $(0.6 \%)$ had a pre-MTB management plan that included clinical trial enrollment, but neither patient was ultimately recommended clinic trial following MTB consensus. Seven prostate cancer patients thought to warrant typical therapies were recommended to enroll in clinical trials following discussion at the MTB.

\section{Discussion}

Our prospective study evaluating a tumour board that discusses all patients with a diagnosis of GU malignancy found a significant proportion (17.8\%) of cancer patients had changes to their treatment plan following multidisciplinary discussion. Moreover, it did not appear that physicians were able to predict which patients would benefit from MTB discussion as those patients who did experience a change in plan were predicted to "likely" have a change $10.5 \%$ of the time versus $8.7 \%$ in the group that did not have a change.

Our results contrast with those of Acher et. al. who performed a similarly designed prospective evaluation of 124 cancer patients discussed at their GU MTB ${ }^{10}$. They found only 2 patients (1.6\%) had a change in management plan following MTB discussion and both patients were among the 10 predicted by providers to likely have a change. This small study concluded that not all "routine" GU oncology patients require discussion at MTB. The MTB in that study consisted of three urologists, a single radiologist, a pathologist, and an oncologist, with no members of radiation oncology present, discussing patients in a community hospital setting. The MTB at our tertiary-care hospital includes at least two oncologists and a pathologist trained specifically to evaluate genitourinary malignancies, a radiation oncologist, and 6 urologists, 
which likely resulted in a greater variety of opinion regarding a more complex population of oncology patients. Additionally, discussion of uptake into clinical trials was not evaluated by Acher et. al.

Our results, indicating a more than 1 in 6 chance of change in treatment plan following MTB discussion, are consistent with literature from other disciplines. A literature review of different MTBs revealed that of 7 studies evaluating for a change in management data, $20 \%$ or more of patients discussed at MTB experienced a change in treatment plan in $5(71 \%)^{12}$. A change in treatment plan following MTB discussion was identified in $20 \%$ of gynecologic cancer patients ${ }^{5}$, $24 \%$ of head and neck cancer patients ${ }^{6}, 52 \%$ of breast cancer patients ${ }^{8}$, and $25.1 \%$ of pancreatic and upper gastrointestinal tract cancer patients ${ }^{9}$, indicating the change in plan in $18 \%$ of GU cancer patients included in our study is consistent with results from other disciplines.

The decision regarding which cancer patients to include in genitourinary MTB meetings varies by institution. Kurpad et al. reported a high degree of change in treatment plan (32.2\%) following MTB review of all GU oncology patients, but their study design included only patients who presented as a referral to their tertiary care center with an outside diagnosis of urologic malignancy. Similarly, Rao et al. reported a high rate (26.7\%) of change following discussion by their institution's GU MTB, which reviews only those cases selected by providers to necessitate multidisciplinary review. Our study is the first to show that discussion of all GU oncology patients in a multidisciplinary setting, regardless of physician assessment of complexity or need, can result in high rates of changes to treatment plans.

The highest rates of plan changes following MTB discussion were identified in bladder cancer (20.6\%), prostate cancer (17.6\%), and upper tract urothelial cancer (33.3\%), with kidney cancer patients being the least likely to experience a change (11.1\%). While multivariable analysis did not reveal these diagnoses to be independently associated with a change, these results seem consistent with practice patterns observed in urology. Most bladder and prostate cancer patients are discussed at MTB following biopsy or transurethral bladder tumour resection (TURBT), whereas kidney cancer patients rarely undergo biopsy and are often presented following a pathologic diagnosis made at the time of definitive treatment (i.e. partial nephrectomy or thermal ablation). Additionally, there is little evidence for adjuvant or neoadjuvant therapies in kidney cancer, limiting the possible treatment options for these patients, but as more therapies are approved for high-risk renal cell carcinoma the likelihood of change in these patients may increase. ${ }^{13}$

Conversely, patients with bladder or prostate cancer have multiple treatment options and adjuvant therapies available to them likely resulting in a high rate of change in these patients ${ }^{14,15}$ Representative examples of changes made in our study include bladder cancer patients who were determined to warrant medical therapy with Bacillus Calmette-Guerin therapy as opposed to surveillance following TURBT and patients with high risk pathologic features following 
prostatectomy who were going to have the option of adjuvant radiation therapy discussed, but were determined by the MTB to be an appropriate candidate for surveillance instead.

Notably, the recommendation of seven prostate cancer patients to be enrolled into clinical trials made up a large proportion of prostate cancer patients who experienced a change in treatment plan (31.8\%), and is reflective of the clinical trials being offered at our center during the study period. This may have resulted in a higher rate of change in the prostate cancer patients. Only two patients $(0.6 \%)$ were recommended to be included in a clinical trial prior to MTB discussion, but neither patient ultimately had a clinical trial as the consensus recommendation by the MTB. This provides evidence that providers at large institutions may not be aware of clinical trials that may be appropriate for their patients, and the MTB may be a good venue to increase inclusion into these important studies. This is consistent with a previous study which suggested there are higher rates of recommendation for clinical trials for GU oncology patients who were included in a teleconferenced MTB. ${ }^{16}$

A physician prediction of a change to be "likely" was also not independently associated (OR 1.03 , 95\% CI 0.34-3.11) with change. Physician perceptions on what may be best for patients or preferred by patients has been questioned in other fields of medicine. Physicians have not been able to reliably predict the odds of patient survival after admission for congestive heart failure or to an intensive care unit. ${ }^{17,18}$ Physicians are unlikely to accurately predict patient preferences regarding decisions on end-of-life care or advanced directives, ${ }^{19,20}$ even in physicians experienced in caring for cancer patients. ${ }^{21}$ Given the results of the literature on physician prediction, it is not surprising the physician prediction of need for discussion at MTB may not be the best criteria for determining which patients should be included.

While cancer type, physician experience, and physician prediction were not shown to be associated with a change in treatment plan following MTB discussion, the initial treatment plan designated prior to MTB meeting was significant. Specifically, patients who were to be given a choice of multiple treatment options (i.e. surveillance versus treatment or a choice of different treatment modalities) were more likely to experience a change in that plan. It can be hypothesized that physicians determining this plan may not feel qualified to remark on the potential benefits or detriments of some treatment options with which they may not be as experienced. Multidisciplinary meetings or clinics with specialists on medical therapies, radiation, and surgery present may be best equipped to determine one therapy may be most specifically suited to an individual patient's circumstances. Uncommon treatment options, when analyzed together for simplicity, were nearly associated with a change in management plan (OR 2.89, 95\%CI 0.88-9.54). Three patients who were thought to require additional workup and two patients who were thought likely to be included in a clinical trial ultimately had typical treatment options decided upon by the MTB, which represented the majority of the changes in this group, and further interpretation of this finding did not seem relevant to discussion. 
Our study has several limitations, including lack of long-term follow-up to assess whether patients did end up receiving or benefiting from recommended therapies. In fact, there is evidence that MTB recommendations are often not followed in practice ${ }^{12}$. Patients were only included in the study if their presenting provider completed the survey prior to MTB discussion, which could result in a sampling bias, especially as the majority of responses were from urology providers. Based on study design, to address our specific hypothesis there was no inclusion of a control group to directly compare our results. Our results from a tertiary referral center may not be generalizable to all centers that care for genitourinary oncology patients.

\section{Conclusion}

A weekly MTB discussion of all genitourinary oncology patients can result in a high rate of change in treatment decision-making and should be considered at any institutions caring for a similar patient population. At institutions where cancer patients are selectively included for MTB discussion, consideration should be given to patients whose are to be offered multiple treatment options and patients whose diagnoses may warrant inclusion in clinical trials being offered at the institution. Physicians could not reliably predict which patients would experience a change in their treatment plan following MTB discussion. 


\section{References}

1. Knutson A, McNamara E. Ensuring Patient-Centered Care. Cancer Progr Stand. 2016. https://www.facs.org/quality-programs/cancer/coc/standards.

2. NCCN. NCCN Clinical Practice Guidelines in Oncology (NCCN Guidelines®) Prostate Cancer. Version 12017. 2017:1-111. www.nccn.org/patients.

3. Kurpad R, Kim W, Rathmell WK, et al. A multidisciplinary approach to the management of urologic malignancies: Does it influence diagnostic and treatment decisions? Urol Oncol Semin Orig Investig. 2011;29(4):378-382. doi:10.1016/j.urolonc.2009.04.008.

4. Sooriakumaran P, Dick JA, Thompson AC, Morley R. The central urology multidisciplinary team - Is it time to change the referral criteria? An audit of practice in a district general hospital in London. Ann R Coll Surg Engl. 2009;91(8):700-702. doi:10.1308/003588409X12486167521190.

5. Greer HO, Frederick PJ, Falls NM, et al. Impact of a weekly multidisciplinary tumour board conference on the management of women with gynecologic malignancies. Int $J$ Gynecol Cancer. 2010;20(8):1321-1325. doi:10.1111/IGC.0b013e3181f5871e.

6. Wheless SA, McKinney KA, Zanation AM. A prospective study of the clinical impact of a multidisciplinary head and neck tumour board. Otolaryngol - Head Neck Surg. 2010;143(5):650-654. doi:10.1016/j.otohns.2010.07.020.

7. Boxer MM, Vinod SK, Shafiq J, Duggan KJ. Do multidisciplinary team meetings make a difference in the management of lung cancer? Cancer. 2011;117(22):5112-5120. doi:10.1002/cncr.26149.

8. Newman EA, Guest AB, Helvie MA, et al. Changes in surgical management resulting from case review at a breast cancer multldisciplinary tumour board. Cancer. 2006;107(10):2346-2351. doi:10.1002/cncr.22266.

9. Brauer DG, Strand MS, Sanford DE, et al. Utility of a multidisciplinary tumour board in the management of pancreatic and upper gastrointestinal diseases: an observational study. Hpb. 2017;19(2):133-139. doi:10.1016/j.hpb.2016.11.002.

10. Acher PL, Young AJ, Etherington-Foy R, McCahy PJ, Deane AM. Improving outcomes in urological cancers: The impact of "multidisciplinary team meetings.” Int J Surg. 2005;3(2):121-123. doi:10.1016/j.ijsu.2005.06.006.

11. Rao K, Manya K, Azad A, et al. Uro-oncology multidisciplinary meetings at an Australian tertiary referral centre - Impact on clinical decision-making and implications for patient inclusion. BJU Int. 2014;114(S1):50-54. doi:10.1111/bju.12764.

12. Lamb BW, Sevdalis N, Mostafid H, Vincent C, Green JSA. Quality Improvement in 
Multidisciplinary Cancer Teams: An Investigation of Teamwork and Clinical DecisionMaking and Cross-Validation of Assessments. Ann Surg Oncol. 2011;18(13):3535-3543. doi:10.1245/s10434-011-1773-5.

13. Cadeddu JA, Chang A, Clark PE, et al. American Urological Association (AUA) Guideline: Renal Mass and Localized Renal Cancer. 2017:1-49.

14. Chang SS, Boorjian SA, Chou R, et al. Diagnosis and Treatment of Non-Muscle Invasive Bladder Cancer: AUA/SUO Guideline. J Urol. 2016;196(4):1021-1029. doi:10.1016/j.juro.2016.06.049.

15. Daneshmand S, Konety BR. American Urological Association ( AUA ) Guideline American Urological Association Non-Muscle Invasive Bladder Cancer. AUA Clin Guidel. 2016;(April):1-45.

16. Bauman G, Winquist E, Chin J. A pilot study of regional participation in a videoconferenced multidisciplinary genitourinary tumour board. Can J Urol. 2005;12(1):2532-2536.

17. Poses RM, Smith WR, McClish DK, et al. Physicians' survival predictions for patients with acute congestive heart failure. Arch Intern Med. 1997;157(9):1001-1007. doi:10.1001/archinte.1997.00440300111009.

18. Rocker G, Cook D, Sjokvist P, et al. Clinician predictions of intensive care unit mortality*. Crit Care Med. 2004;32(5):1149-1154. doi:10.1097/01.CCM.0000126402.51524.52.

19. Wenger NS, Phillips RS, Teno JM, et al. Physician Understanding Of Patient Resuscitation Preferences: Insights and Clinical Implications. J Am Geriatr Soc. 2000;48(S1):S44-S51. doi:10.1111/j.1532-5415.2000.tb03140.x.

20. Fischer GS, Tulsky J a, Rose MR, Siminoff L a, Arnold RM. Patient Knowledge and Physician Predictions of Advance Directives. Knowl Creat Diffus Util. 1998;13(7):447454. http://www.ncbi.nlm.nih.gov/pubmed/9686710.

21. Bruera E, Sweeney C, Calder K, Palmer L, Benisch-Tolley S. Patient Preferences Versus Physician Perceptions of Treatment Decisions in Cancer Care. J Clin Oncol. 2001;19(11):2883-2885. doi:10.1200/JCO.2001.19.11.2883. 
Figures and Tables

\begin{tabular}{|c|c|c|c|c|}
\hline Characteristic & $\begin{array}{c}\text { No change } \\
(n=264)\end{array}$ & $\begin{array}{l}\text { Change } \\
(n=57)\end{array}$ & $\begin{array}{c}\text { Total } \\
(n=321)\end{array}$ & $\mathbf{p}$ \\
\hline \multicolumn{5}{|l|}{ Sex, n (\%) } \\
\hline Male & $220(81.2)$ & $51(18.8)$ & $271(84.7)$ & 0.25 \\
\hline Female & $44(88.0)$ & $6(12.0)$ & $50(15.6)$ & \\
\hline \multicolumn{5}{|l|}{ Age, n (\%) } \\
\hline$\leq 60$ & 85 (86.7) & $13(13.3)$ & $98(30.5)$ & 0.24 \\
\hline $61-75$ & $121(80.7)$ & 29 (19.3) & $150(46.7)$ & \\
\hline$>75$ & $58(79.5)$ & $15(20.6)$ & $73(22.7)$ & \\
\hline \multicolumn{5}{|l|}{ Cancer type, n (\%) } \\
\hline Kidney & 56 (88.9) & 7 (11.1) & $63(19.6)$ & 0.26 \\
\hline Bladder & $81(79.4)$ & $21(20.6)$ & $102(31.8)$ & \\
\hline Prostate & $103(82.4)$ & $22(17.6)$ & $125(38.9)$ & \\
\hline Testicle & $7(87.5)$ & $1(12.5)$ & $8(2.5)$ & \\
\hline UTUC & $8(66.7)$ & $4(33.3)$ & $12(3.7)$ & \\
\hline Other & $9(81.8)$ & $2(18.2)$ & $11(3.4)$ & \\
\hline \multicolumn{5}{|l|}{ Specialty, n (\%) } \\
\hline Urology & $252(82.1)$ & 55 (17.9) & 307 (95.6) & 0.64 \\
\hline Med-oncology & $8(80.0)$ & $2(20.0)$ & $10(3.1)$ & \\
\hline Rad-oncology & $4(100)$ & $0(0)$ & $4(1.3)$ & \\
\hline \multicolumn{5}{|l|}{$\begin{array}{l}\text { Years in practice, } \\
n(\%)\end{array}$} \\
\hline$<5$ years & $100(86.2)$ & $16(13.8)$ & $116(36.1)$ & 0.16 \\
\hline$\geq 5$ years & $164(80.0)$ & $41(20.0)$ & 205 (63.9) & \\
\hline \multicolumn{5}{|l|}{$\begin{array}{l}\text { Fellowship training, } \\
\mathrm{n}(\%)\end{array}$} \\
\hline No & $76(81.7)$ & 17 (18.3) & $93(30.3)$ & 0.91 \\
\hline Yes & $176(82.2)$ & $38(17.8)$ & $214(69.7)$ & \\
\hline \multicolumn{5}{|l|}{$\begin{array}{l}\text { Provider prediction, } n \\
(\%)\end{array}$} \\
\hline $\begin{array}{l}\text { Very unlikely to } \\
\text { change }\end{array}$ & $126(85.7)$ & $21(14.3)$ & $147(45.8)$ & 0.18 \\
\hline Unlikely to change & 115 (79.3) & $30(20.7)$ & $145(45.2)$ & \\
\hline Likely to change & $23(79.3)$ & $6(20.7)$ & $29(9.0)$ & \\
\hline
\end{tabular}




\begin{tabular}{|l|c|c|c|c|}
\hline $\begin{array}{l}\text { Pre-TB physician } \\
\text { plan }\end{array}$ & & & & $<0.01$ \\
\hline Surveillance & $107(87.0)$ & $16(13.0)$ & $123(38.3)$ & \\
\hline Surgery & $27(90.0)$ & $3(10.0)$ & $30(9.4)$ & \\
\hline Medical & $40(90.9)$ & $4(9.1)$ & $44(13.7)$ & \\
\hline Radiation & $9(81.8)$ & $2(18.2)$ & $11(3.4)$ & \\
\hline Discuss options & $69(72.6)$ & $26(27.4)$ & $95(29.6)$ & \\
\hline Other & $12(66.7)$ & $6(33.3)$ & $18(5.6)$ & \\
\hline
\end{tabular}

UTUC: upper tract urothelial carcinoma.

\begin{tabular}{|c|c|c|c|c|c|c|}
\hline & Univa & te analysis & & Multi & ate analysis & \\
\hline Characteristic & OR & $95 \%$ CI & $\mathbf{p}$ & OR & 95\% CI & $\mathbf{p}$ \\
\hline \multicolumn{7}{|l|}{ Sex } \\
\hline Male & 1.00 & Referent & & 1.00 & Referent & \\
\hline Female & 0.59 & $0.24-1.46$ & 0.25 & 0.56 & $0.20-1.59$ & 0.28 \\
\hline \multicolumn{7}{|l|}{ Age } \\
\hline$\leq 60$ & 0.64 & $0.31-1.30$ & 0.22 & 0.93 & $0.42-2.06$ & 0.85 \\
\hline $61-75$ & 1.00 & Referent & & 1.00 & Referent & \\
\hline$>75$ & 1.08 & $0.54-2.17$ & 0.83 & 0.92 & $0.41-2.05$ & 0.83 \\
\hline \multicolumn{7}{|l|}{ Cancer type } \\
\hline Kidney & 1.00 & Referent & & 1.00 & Referent & \\
\hline Bladder & 2.07 & $0.83-5.21$ & 0.12 & 1.7 & $0.58-4.96$ & 0.33 \\
\hline Prostate & 1.70 & $0.69-4.24$ & 0.25 & 0.72 & $0.24-2.15$ & 0.55 \\
\hline Testicle & 1.14 & $0.12-10.71$ & 0.91 & 0.87 & $0.07-10.76$ & 0.91 \\
\hline UTUC & 4.00 & $0.95-16.79$ & 0.06 & 3.99 & $0.70-22.84$ & 0.12 \\
\hline Other & 1.78 & $0.32-9.94$ & 0.51 & 1.24 & $0.19-8.01$ & 0.82 \\
\hline \multicolumn{7}{|l|}{ Specialty } \\
\hline Urology & 1.31 & $0.28-6.02$ & 0.73 & & & \\
\hline $\begin{array}{l}\text { Oncology or } \\
\text { radiation }\end{array}$ & 1.00 & Referent & & & & \\
\hline \multicolumn{7}{|l|}{ Years in practice } \\
\hline$<5$ years & 0.69 & $0.34-1.20$ & 0.16 & 0.55 & $0.25-1.20$ & 0.13 \\
\hline
\end{tabular}




\begin{tabular}{|l|c|c|c|c|c|c|}
\hline$\geq 5$ years & 1.00 & Referent & & 1.00 & Referent & \\
\hline Fellowship training & & & & & & \\
\hline No & 1.00 & Referent & & 1.00 & Referent & \\
\hline Yes & 0.97 & $0.51-1.82$ & 0.91 & 1.17 & $0.50-2.74$ & 0.72 \\
\hline Provider prediction & & & & & & \\
\hline $\begin{array}{l}\text { Very unlikely to } \\
\text { change }\end{array}$ & 0.64 & $0.35-1.18$ & 0.15 & 0.63 & $0.30-1.34$ & 0.23 \\
\hline Unlikely to change & 1.00 & Referent & & 1.00 & Referent & \\
\hline Likely to change & 1.00 & $0.37-2.68$ & 1.00 & 0.86 & $0.26-2.82$ & 0.80 \\
\hline $\begin{array}{l}\text { Pre-TB treatment } \\
\text { plan }\end{array}$ & & & & & & \\
\hline Surveillance & 1.00 & Referent & & 1.00 & Referent & \\
\hline Surgery & 0.74 & $0.20-2.74$ & 0.66 & 0.51 & $0.13-2.08$ & 0.35 \\
\hline Medical & 0.67 & $0.21-2.12$ & 0.49 & 0.34 & $0.09-1.33$ & 0.12 \\
\hline Radiation & 1.49 & $0.29-7.51$ & 0.63 & 2.47 & $0.43-14.29$ & 0.31 \\
\hline Discuss options & 2.52 & $1.26-5.03$ & 0.01 & 2.41 & $1.05-5.49$ & 0.04 \\
\hline Other & 3.34 & $1.10-10.17$ & 0.03 & 4.00 & $1.13-14.16$ & 0.03 \\
\hline
\end{tabular}

CI: confidence interval; OR: odds ratio; UTUC: upper tract urothelial carcinoma.

\begin{tabular}{|l|l|l|l|l|l|l|l|}
\hline \multicolumn{2}{|l|}{ Table 3. Most common treatment change outcomes } & \multicolumn{2}{l|}{} & \\
\hline $\begin{array}{l}\text { Physician } \\
\text { prediction }\end{array}$ & TB outcome & Prostate & Bladder & Kidney & UTUC & Other & All \\
\hline Discuss options & Medical therapy & 1 & 3 & 1 & 3 & 1 & 9 \\
\hline Surveillance & Medical therapy & 1 & 6 & 2 & 0 & 0 & 9 \\
\hline Discuss options & Clinical trial & 5 & 0 & 0 & 0 & 0 & 5 \\
\hline Discuss options & Surveillance & 4 & 0 & 0 & 1 & 0 & 5 \\
\hline Discuss options & Surgery & 2 & 1 & 0 & 0 & 0 & 3 \\
\hline Surgery & Surveillance & 0 & 2 & 0 & 0 & 0 & 2 \\
\hline Discuss options & Multimodal & 0 & 2 & 0 & 0 & 0 & 2 \\
\hline Clinical trial & therapy & & & & & & \\
\hline Surveillance & Surgery & 0 & 0 & 1 & 0 & 1 & 2 \\
\hline Surveillance & Workup & 1 & 1 & 2 & 0 & 0 & 2 \\
\hline Other & & 8 & 6 & 1 & 0 & 1 & 16 \\
\hline Total & & 22 & 21 & 7 & 4 & 3 & 57 \\
\hline
\end{tabular}

UTUC: upper tract urothelial carcinoma. 